\title{
Isolamenti di Salmonella spp. da matrici ambientali nella regione Molise negli anni 2003-2005
}

\author{
Carla Pepe, Rita De Socio, Antonella Melloni, Luciana Di Pardo, Annamaria Manuppella \\ ARPA Molise - Dipartimento Provinciale di Isernia; via Giovanni Berta $n^{\circ}$ I - 86170 Isernia
}

Key words: Enter-Net / Salmonella spp./serotyping.

Isolation of Salmonella spp. from environmental matrices in Molise region in 2003-2005

\section{SUMMARY}

Enter-Net is a European network used for the surveillance of human gastrointestinal infections. This network was created in 1994 and is coordinated by the HPA in Colindale. In Italy, this network is managed by the Superior Health Institute and its participants include various referral centres present in most of the regions in Italy. In Molise, the centre is located at the Arpa Provincial Department of Isernia. This centre receives strains of isolated Salmonella from six Regional hospital laboratories. In addition, the centre receives strains from other Arpa laboratories originating from surface and sewer water. All of the Centre's activities are conducted according to the Italian law (D.L.vo $n^{\circ}$ 152/99). The isolates are tested for serotyping classification and antimicrobial susceptibility. During the three year period (2003-2005), 228 Salmonella strains were isolated throughout the principle water basins. $18.9 \%$ of the isolates were from the Biferno basins, in which the water is considered drinkable, while $51.7 \%$ were from various water bodies flowing into the Molise costal areas. Consequently, the potential impact on drinkable and recreational water is rather consistent. Particularly considering that 26 isolates (22.1\%) belong to serotypes relevant for public health (S.typhimurium, S.enteritidis, S.hadar, S.infantis, S.virchow). The results confirmed the presence of these endemic serotypes in surface waters in Molise. The results have also showned the impact that these could have on public health.

\section{INTRODUZIONE}

Nella Regione Molise la sorveglianza dei patogeni enterici si svolge ad opera di un Centro di Riferimento Regionale ubicato presso il Dipartimento di Isernia dell'ARPA Molise.

Il Centro opera in stretta collaborazione con l'Istituto Superiore di Sanità (ISS), Centro di Riferimento Nazionale nell'ambito del sistema ENTER - NET, il network europeo per la sorveglianza di Salmonella spp. e di E.coli verocitotossico $(4,5,9,10)$.

Al Dipartimento di Isernia pervengono gli stipiti di Salmonella spp. e di altri patogeni enterici (Campylobacter spp. ed Aeromonas spp.) isolati dai laboratori di microbiologia clinica dei sei ospedali regionali, nonché gli stipiti di salmonella isolati da acque superficiali e di scarico. Gli stipiti vengono sierotipizzati e viene saggiata la sensibilità agli antibiotici.

I ceppi di provenienza ambientale vengono isolati dai Dipartimenti ARPA nell'ambito delle attività di monitoraggio e controllo previste dal D.L.vo $\mathrm{n}^{\circ} 152 / 99$ e s.m.i. (8); il Decreto impone la ricerca di Salmonella spp. solo dalle acque destinate alla produzione di acqua potabile, mentre per le altre tipologie è contemplata la determinazione di E.coli; tuttavia questo microrganismo, il principale fra gli indicatori di inquinamento fecale, non sempre è direttamente correlato alla presenza delle salmonelle che, specie nelle acque reflue, risultano più resistenti ai trattamenti di depurazione $(2,6$, ) e a volte si riscontrano anche in concomitanza con una bassa colimetria(11).

Risulta importante, pertanto, individuare nelle acque $i$ patogeni enterici, ed in primo luogo Salmonella spp., che è il più frequente agente eziologico di malattia gastroenterica acuta nell'uomo, sia in Italia che in Molise ( 2, 4, 5, 6, 9, $10,14,15,19,20,21,22)$.

Difatti l'ambiente può rappresentare un reservoir per molti sierotipi di salmonella che sopravvivono nelle acque reflue, contaminando suolo ed alimenti di origine vegetale; in particolare il riutilizzo dei liquami a scopo agricolo $(3,7)$, pratica molto diffusa anche in Italia, può comportare rischio di contaminazioni specialmente per le verdure a foglie da consumare crude (2).

Un'altra importante modalità di diffusione riguarda gli ambienti acquatici (torrenti, fiumi, mari e laghi); dopo l'immissione degli effluenti in tali ambienti, la concentrazione dei microrganismi si riduce per effetto di vari fattori (diluizione, temperatura, irraggiamento, etc); tuttavia, malgrado ciò, è stato dimostrato che l'immissione dei reflui comporta l'incremento della diffusione di virus enterici e di salmonella nei corpi idrici ricettori, 
compromettendo il loro utilizzo a scopo ricreazionale $(2,6,10)$ e potabile.

Nel Molise l'orografia del territorio consente l'esistenza di un fitto reticolo idrografico, nell'ambito del quale si riscontrano numerosi bacini imbriferi: Trigno, Volturno, Saccione, Fortore, Sinarca, Tecchio, Biferno. Quest'ultimo bacino soddisfa le richieste di acqua potabile di tutto il basso Molise attraverso l'invaso artificiale del Liscione, originato da uno sbarramento sull'asta fluviale principale; l'invaso di Occhito, generato da uno sbarramento sul Fortore, serve a scopo potabile per la regione Puglia.

Sulla scorta di tali considerazioni si ritenuto opportuno, nel presente lavoro, descrivere l'andamento della diffusione ambientale di Salmonella spp nel Molise negli anni 2003 - 2005, onde valutarne il potenziale ruolo nella trasmissione di infezioni enteriche.

\section{MATERIALI E METODI}

Gli stipiti ambientali di Salmonella sono stati isolati dalle acque superficiali e reflue secondo il metodo APAT - IRSA (1); per ogni campione è stata prelevata un'aliquota da un litro in un recipiente di vetro pyrex sterilizzato, contenente sodio tiosolfato per la neutralizzazione del cloro eventualmente presente. L'esame è stato effettuato entro le 24 ore dal prelievo, filtrando l'acqua attraverso membrane filtranti sterili di acetato di cellulosa da 0,45 micron; i terreni utilizzati sono stati: Acqua Peptonata Tamponata, Brodo Rappaport Vassiliadis, Hektoen Enteric agar, Kligler Iron Agar, Lysin Iron Agar. Le colonie sospette sono state identificate biochimicamente tramite il sistema miniaturizzato API $20 \mathrm{E}$.

La tipizzazione sierologica è stata condotta seguendo le procedure standard dello schema di Kauffmann - Withe (16); le colture sono state saggiate prima con antisieri polivalenti, per l'individuazione dell'antigene somatico, e poi con i monovalenti facenti parte del pool di antigeni compreso nel polivalente risultato positivo. Una volta individuato l'antigene "O", si è proceduto alla definizione della coppia di antigeni " $H$ " caratterizzanti i sierotipi; a tal fine è stato utilizzato il metodo "Sven Gard", consistente nella semina puntiforme del ceppo di salmonella al centro di una piastra petri contenente agar molle; dopo 24 ore di incubazione, la patina microbica migrata alla periferia della piastra, particolarmente ricca di antigeni flagellari, è stata testata su vetrino con antisieri diagnostici. Poiché la maggioranza delle salmonelle è difasica, (possiede cioè due fasi antigeniche $\mathrm{H}$, chiamate fase $1 \mathrm{e}$ fase 2), al momento della prima agglutinazione " $\mathrm{H}$ " si è evidenziata solo la fase prevalente; è stato per- ciò necessario inibire tale fase addizionando all'agar molle gli specifici antisieri ad alto titolo anticorpale corrispondenti alla fase prevalente. Dopo incubazione a $37^{\circ} \mathrm{C}$ per $24 \mathrm{~h}$, i ceppi sono stati testati per l'individuazione della fase mancante.

Dalla consultazione dello schema di KauffmannWhite si è così individuato il sierotipo di Salmonella corrispondente alla combinazione degli antigeni "O" e di quelli " $\mathrm{H}$ " delle due fasi individuate (24).

Gli antibiogrammi sono stati effettuati secondo il metodo di Kirby Bauer (24) su piastre di agar Isosensitest; gli antibiotici testati, previsti dal protocollo ENTER - NET, sono: Acido Nalidixico (NA), Ampicillina (AM), Cefotaxime (CTX), Ciprofloxacina (CIP), Cloramfenicolo (C), Gentamicina (GM), Kanamicina (K), Streptomicina (S), Sulfisoxazolo (S3), Tetraciclina (Te), Trimethoprim (TMP). Cefalotina (KF), Amoxicillina/Acido Clavulanico (AMC) e TMP/Sulfamethoxazole (SXT) sono stati introdotti nel panel di antibiotici da testare solo dal 2004.

Periodicamente (ogni 10 tests circa) è stato effettuato un controllo della qualità della procedura utilizzando un ceppo di E. coli ATCC 25922.

\section{RISULTATI}

Nella Regione Molise nel triennio considerato sono stati isolati 242 stipiti di Salmonella spp., in prevalenza (mediamente nel $94 \%$ dei casi) da acque superficiali (tabella1), la cui caratterizzazione sierologica è riportata in tabella 2 .

Dall'osservazione dei dati si rileva una notevole variabilità di sierotipi, superiore a quella riscontrata in campo umano (17); S.typhimurium nel 2003 e 2004 è stata isolata dall'ambiente con maggiore frequenza rispetto agli altri sierotipi, come peraltro è avvenuto anche per gli isolamenti umani; questo sierotipo è stato quello più frequentemente isolato dall'uomo già dal 2000, anno in cui un suo particolare clone causò in Provincia di Isernia una tossinfezione alimentare provocata dall'ingestione di insaccati contaminati; in quella circostanza tutti i ceppi isolati da malati, dall'alimento e dalle acque superficiali furono caratterizzati dal punto di vista molecolare tramite elettroforesi pulsata dall'ISS, che confermò l'appartenenza di tutti gli stipiti al medesimo genotipo (12).

Nel 2004 per la prima volta in Molise è stata isolata S.veneziana, che in Italia rappresenta il secondo sierotipo più frequentemente isolato $\mathrm{da}$ campioni ambientali (nella quasi totalità da acque dolci superficiali), interessando in particolare le regioni bagnate dal mare Adriatico; anche il sierotipo monofasico 4, (5), 12: i:-, la cui diffusione in Italia e in Europa durante l'ultimo decennio ha 
subito un costante aumento $(4,5)$, è sempre presente nelle acque superficiali molisane, rappresentando mediamente il $3.1 \%$ degli isolamenti. Tale tipo sierologico, apparentemente simile a S.typhimurium (formula antigenica 4, (5), 12: i: 1, 2 ), è stato individuato recentemente come clone a sé stante, geneticamente diverso dal predetto serovar ed è stato collegato ai suini ed agli alimenti a base di carne di maiale $(4,5,10)$.

Nel 2005 "S.derby" è stato il sierotipo isolato con maggiore frequenza, mentre S.enteritidis, secondo sierotipo più frequente nei casi umani in Molise ed in Italia $(4,5,10)$, nei campioni ambientali molisani è stato isolato solo nel $2003 \mathrm{e}$ 2005 con uno scarso numero di ceppi.

Tale circostanza è spiegabile se si considera l'epidemiologia delle infezioni da S.enteritidis; questo sierotipo, infatti, presenta una notevole specificità d'ospite, essendo fortemente adattato alle specie avicole. S.typhimurium, invece, molto più frequente in campo ambientale, è un germe ubiquitario, in grado di colonizzare l'ambiente ed utilizzare come reservoir un'ampia gamma di specie animali (13). Va rilevato, inoltre, il riscontro negli anni considerati di 20 sierotipi ( $8.3 \%$ sul totale) ascrivibili alle sottospecie II, III e IV il cui comportamento epidemiologico non è ben noto; da studi fatti risulta che animali selvatici come la volpe, insieme ad altri animali non mammiferi (lucertola) o a sangue freddo, possano giocare un ruolo nel mantenimento nell'ambiente di tali serovars, peraltro di difficile riscontro nell'uomo e negli alimenti di origine animale, indicando solo un ciclo naturale nell'ambiente che non coinvolge la sanità pubblica (23).

Di contro, fra gli isolamenti relativi a tutti gli anni considerati, si riscontrano gli stipiti individuati dalla Commissione Europea come rilevanti per la salute pubblica: S.typhimurium, S.enteritidis, S.hadar, S.infantis e S.virchow (Reg.1003/2005/CE)(18).

Tali sierotipi, considerati cumulativamente, rappresentano per gli anni 2003, 2004 e 2005 rispettivamente il $35.1 \%$, il $25.4 \%$ e il $18.8 \%$ degli isolati, e sono stati rilevati in prevalenza presso il bacino del Volturno (21 isolamenti), seguito dal Biferno, dal Trigno, dal Sangro (9 isolamenti per ciascun bacino), dai bacini minori (Saccione, Rio Vivo, Sinarca e Tecchio, con 4 isolamenti) e Fortore (1 isolamento).

Andando a considerare la totalità degli stipiti iso- lati da bacini che recapitano in mare (118 ceppi, pari al $51.7 \%$ degli isolamenti), nel tratto di costa che interessa la Regione Molise (Biferno, Trigno, Saccione, Rio Vivo, Sinarca, Tecchio si rileva un impatto potenziale sulle acque di balneazione piuttosto consistente, specie se si considera che 26 sierotipi, pari al $22.1 \%$ di tali stipiti, rientrano nel set di quelli rilevanti per la salute pubblica. Per quanto attiene all'utilizzo potabile delle acque, il rischio di contaminazione è più consistente per il bacino del Biferno, presso il quale sono stati isolati 43 stipiti (18.9\% rispetto al totale), 9 dei quali compresi nel set sopra menzionato.

La distribuzione temporale degli isolamenti presenta il tipico andamento stagionale, con valori massimi nei mesi estivi e nel periodo autunnoinvernale, il che rispecchia l'ecologia tipica del germe (Figure I - II ).

Il fenomeno della resistenza ha riguardato il $68 \%$ dei ceppi di salmonella nel 2003, il 73.5\% nel 2004 e il $64.3 \%$ nel 2005. In particolare S.typhimurium ha manifestato elevate percentuali di resistenza soprattutto nei confronti di Ampicillina, Sulfisoxazolo, Streptomicina e Tetraciclina, come peraltro avviene in campo umano (Figura III). Nel 2004 questo sierotipo ha mostrato resistenza in maniera piuttosto marcata anche al Cloramfenicolo.

Anche la multiresistenza ha riguardato principalmente questo sierotipo (Figura IV), con resistenze multiple fino a 7 antibiotici; l'associazione prevalente è stata: Ampicillina - Cloramfenicolo Streptomicina - Sulfisoxazolo - Tetraciclina Amoxicillina/Acido Clavulanico (ACSSuTAMC: 22.7\%), seguita da Ampicillina, Streptomicina, Sulfisoxazolo, Tetraciclina (ASSuT: 18.2\%). Quest'ultimo pattern di multiresistenza è stato posto in relazione con fagotipi presenti nell'uomo e nei suini $(4,5,10,13,17)$.

Nel 2005 S.derby, il serovar più frequentemente isolato, ha mostrato una resistenza dell' $84.6 \%$ (Figura V) mentre la multiresistenza, a partire da tre antibiotici, è stata dell' $81.8 \%$ e l'associazione prevalente è stata: S-S3-Te (63.6\%). Il secondo sierotipo nel 2005 è risultato essere S.newport che non ha mostrato alcuna resistenza, mentre S.typhimurium è risultata resistente per $1^{\prime} 87.5 \%$ con una multiresistenza, a partire da quattro antibiotici, del $71.4 \%$.

Tabella I. Isolamenti di Salmonella spp. da matrici ambientali. Anni 2003 - 2004-2005.

\begin{tabular}{lcccccc}
\hline Matrice & $\mathbf{2 0 0 3}$ & \multicolumn{2}{c}{$\mathbf{2 0 0 4}$} & \multicolumn{2}{c}{$\mathbf{2 0 0 5}$} \\
\hline Acque superficiali & $\mathbf{n}^{\circ}$ & $\%$ & $\mathbf{n}^{\circ}$ & $\%$ & $\mathbf{n}^{\circ}$ & $\%$ \\
\hline Acque reflue & 71 & 96.0 & 63 & 94.0 & 94 & 93.0 \\
\hline Altro (sedimenti) & 3 & 4.0 & 4 & 6.0 & 6 & 6.0 \\
\hline TOTALE & & & & & 1 & 1.0 \\
\hline
\end{tabular}


Tabella II. Sierotipi di Salmonella spp. isolati dall'ambiente. Anni 2003 - 2004- 2005.

\begin{tabular}{|c|c|c|c|c|c|c|c|c|}
\hline \multicolumn{3}{|l|}{2003} & \multicolumn{3}{|l|}{2004} & \multicolumn{3}{|l|}{2005} \\
\hline Sierotipo & $n^{\circ}$ & $\%$ & Sierotipo & $n^{\circ}$ & $\%$ & Sierotipo & $n^{\circ}$ & $\%$ \\
\hline Typhimurium & 13 & 17.56 & Typhimurium & 10 & 14.92 & Derby & 13 & 12.87 \\
\hline Infantis & 6 & 8.11 & Sott. II 42:z: I,5 & 7 & 10.44 & Newport & 10 & 9.90 \\
\hline Sott. II 42:z; I.5 & 5 & 6.76 & Rissen & 6 & 8.95 & Typhimurium & 8 & 7.92 \\
\hline Livingstone & 5 & 6.76 & Sott. IV 43:z4z23 & 4 & 5.97 & Infantis & 7 & 6.93 \\
\hline Newport & 4 & 5.41 & Hadar & 4 & 5.97 & Sott. II 42:z: I,5 & 6 & 5.94 \\
\hline Goldcoast & 4 & 5.41 & Newport & 3 & 4.47 & Sott. II 4I:z: I,5 & 5 & 4.95 \\
\hline Hadar & 4 & 5.41 & Infantis & 3 & 4.47 & Sott. III b 38:I,v:z35 & 5 & 4.95 \\
\hline Rissen & 3 & 4.05 & Derby & 3 & 4.47 & Sott. IV 43:z4z23 & 5 & 4.95 \\
\hline Panama & 3 & 4.05 & London & 3 & 4.47 & Thompson & 4 & 3.96 \\
\hline Heidelberg & 3 & 4.05 & Szentes & 3 & 4.47 & Sott. III a 48:z4z23 & 4 & 3.96 \\
\hline Thompson & 3 & 4.05 & Thompson & 2 & 2.98 & Napoli & 3 & 2.97 \\
\hline $4,5: \mathrm{i}:-$ & 3 & 4.05 & Anatum & 2 & 2.98 & 4,12:i:- & 3 & 2.97 \\
\hline Kottbus & 2 & 2.70 & Mbandaka & $\mathrm{I}$ & 1.49 & Bredeney & 2 & 1.98 \\
\hline Virchow & 2 & 2.70 & Kunduchi & $\mathrm{I}$ & 1.49 & Rissen & 2 & 1.98 \\
\hline Enteritidis & $\mathbf{I}$ & 1.35 & $4,5,12: \mathrm{i}-$ & 1 & 1.49 & Livingstone & 2 & 1.98 \\
\hline Sott. IV 40:z4z23 & $\mathrm{I}$ & 1.35 & Bovismorbificans & $\mathrm{I}$ & 1.49 & Hadar & 2 & 1.98 \\
\hline Brandenburg & $\mathrm{I}$ & 1.35 & Sott. III b 60:r:z & $\mathrm{I}$ & 1.49 & Blockley & 2 & 1.98 \\
\hline Senftenberg & $\mathrm{I}$ & 1.35 & Livingstone & $\mathrm{I}$ & 1.49 & Goldcoast & 1 & 0.99 \\
\hline Blockley & 1 & 1.35 & Brandenburg & 1 & 1.49 & Enteritidis & $\mathbf{I}$ & 0.99 \\
\hline Bracknell & $\mathrm{I}$ & 1.35 & Worthington & 1 & 1.49 & Brandenburg & 1 & 0.99 \\
\hline Derby & $\mathrm{I}$ & 1.35 & Kottbus & 1 & 1.49 & Anatum & 1 & 0.99 \\
\hline Atzeca & $\mathrm{I}$ & 1.35 & Sott. III b 50:z52:z53 & 1 & 1.49 & Virchow & $\mathbf{I}$ & 0.99 \\
\hline Bovismorbificans & $\mathrm{I}$ & 1.35 & Westhampton & 1 & 1.49 & Panama & 1 & 0.99 \\
\hline Sott. II 6,7: z:z42 & $\mathrm{I}$ & 1.35 & Indiana & 1 & 1.49 & Kapemba & 1 & 0.99 \\
\hline Montevideo & 1 & 1.35 & Kedougou & 1 & 1.49 & Kimuenza & 1 & 0.99 \\
\hline Agona & 1 & 1.35 & Muenster & 1 & 1.49 & Sanktjohann & 1 & 0.99 \\
\hline Anatum & $\mathrm{I}$ & 1.35 & Veneziana & $\mathrm{I}$ & 1.49 & Senftenberg & 1 & 0.99 \\
\hline \multirow[t]{8}{*}{ Kedougou } & 1 & 1.35 & Sott. IV 40:z4z23 & 1 & 1.49 & Corvallis & 1 & 0.99 \\
\hline & & & Sott. II 42:I,v:enz I 5 & $\mathrm{I}$ & 1.49 & 4,5,I2:i:- & 1 & 0.99 \\
\hline & & & & & & Sott. II 42:z: z6 & 1 & 0.99 \\
\hline & & & & & & Sott. II 43:z: I,5 & $\mathrm{I}$ & 0.99 \\
\hline & & & & & & Sott. III b |4:r:z & 1 & 0.99 \\
\hline & & & & & & Sott. III b 50:k:I,5,7 & 1 & 0.99 \\
\hline & & & & & & Sott.III b 50:z52:z35 & 1 & 0.99 \\
\hline & & & & & & Sott IV 38:z4z23 & 1 & 0.99 \\
\hline
\end{tabular}

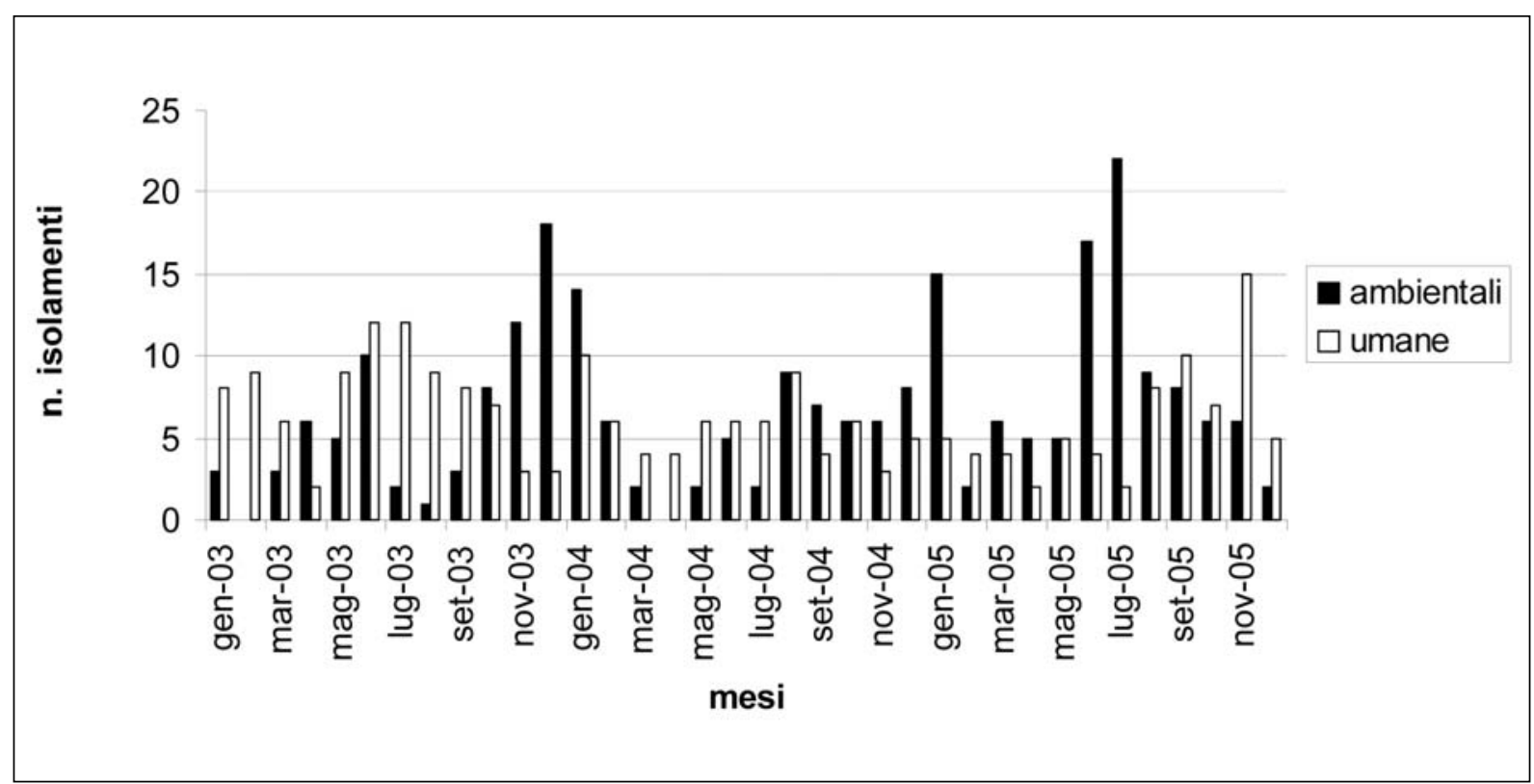

Figura I. Distribuzione mensile degli isolamenti di Salmonella spp. dall'uomo e dall'ambiente. Anni 2003 - 2004 - 2005. 


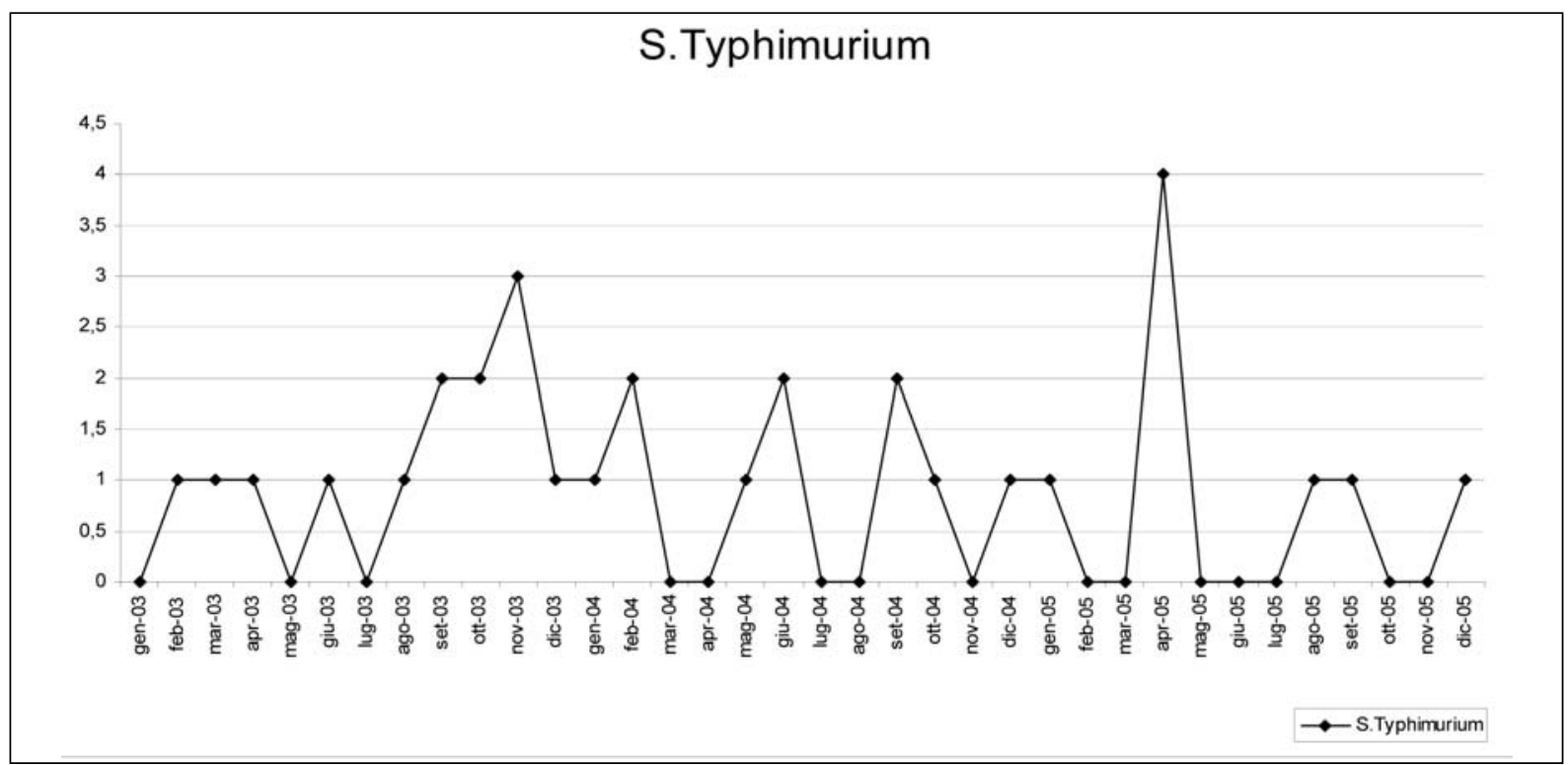

Figura II. Distribuzione temporale S. Typhimurium. Anni 2003 - $2004-2005$.

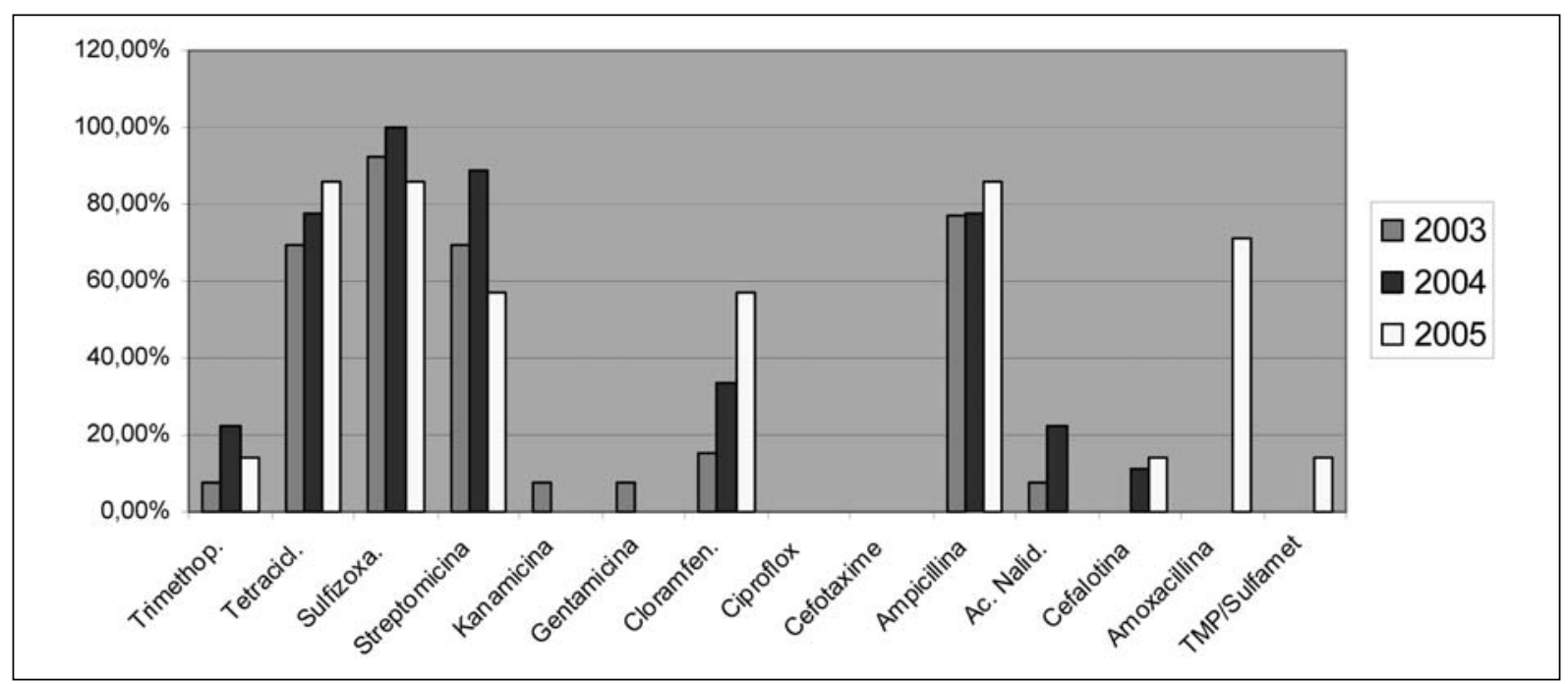

Figura III. \% Resistenza agli antibiotici S. Typhimurium. Anni 2003 - 2004 - 2005.

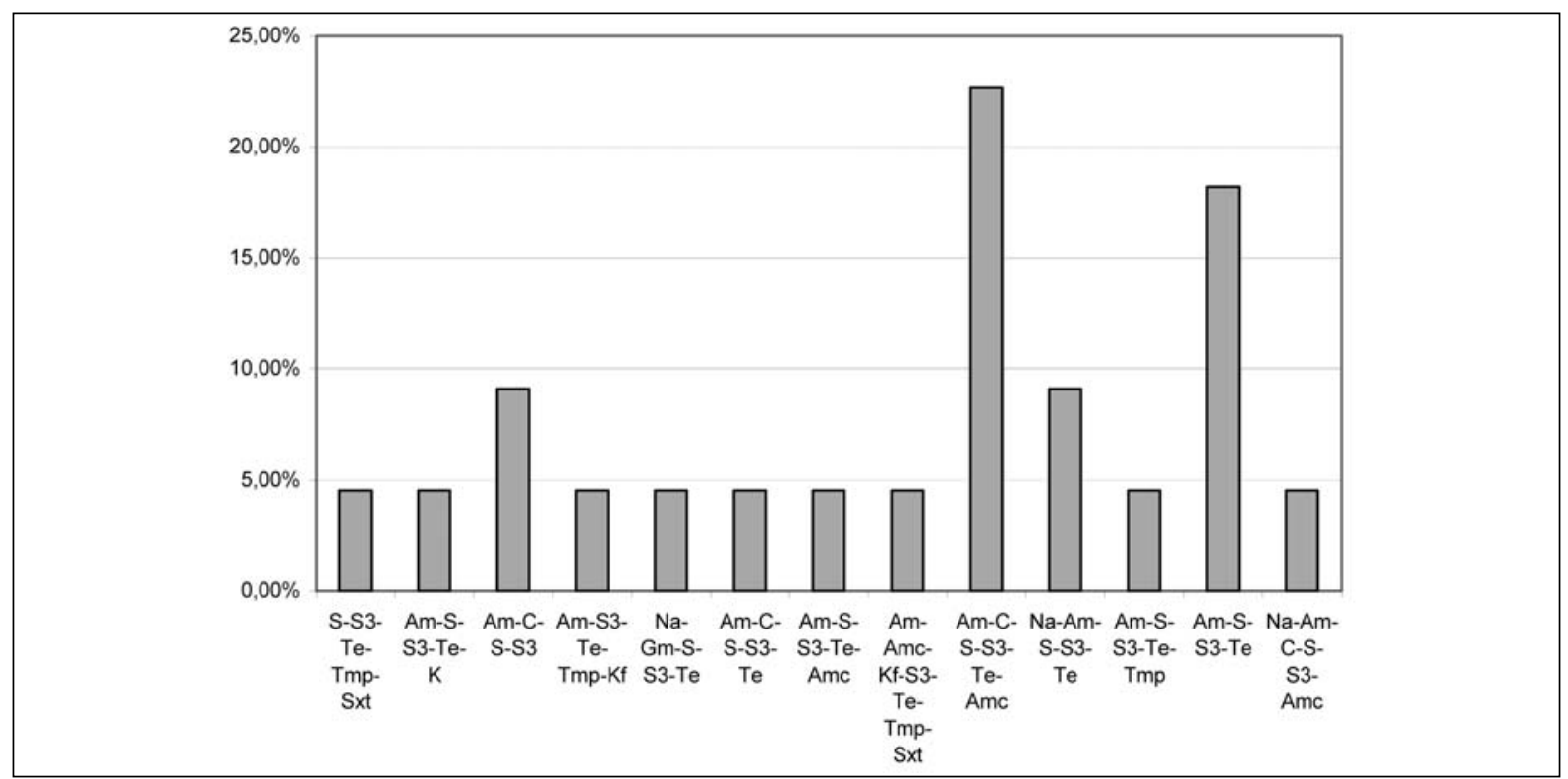

Figura IV. \% Multiresistenza agli antibiotici S. Typhimurium. Anni 2003-2004-2005. 


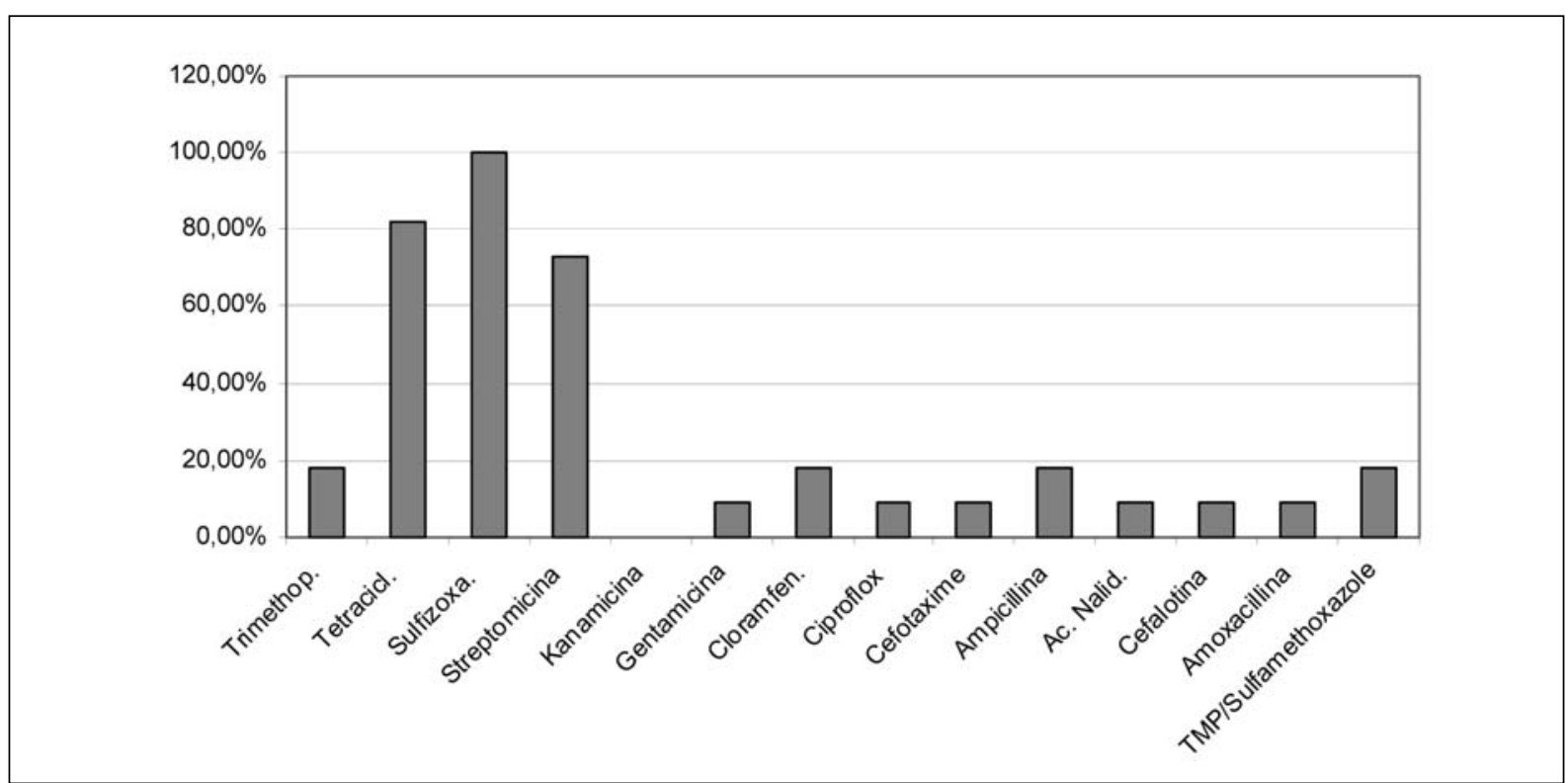

Figura V. \% Resistenza agli antibiotici S. Derby. Anno 2005.

\section{CONCLUSIONI}

Il sistema Enter-Net rappresenta un esempio concreto e positivo di sorveglianza poiché permette, attraverso un database comune, lo scambio di informazioni tra i diversi Paesi che partecipano al progetto consentendo in tal modo di utilizzare $\mathrm{i}$ risultati per studiare l'epidemiologia delle infezioni da Salmonella spp e programmare così eventuali misure di prevenzione e di controllo. La conoscenza di quanto avviene a livello delle matrici ambientali, con particolare riferimento al controllo della contaminazione microbiologica dei corpi idrici e alle caratteristiche dei reflui, matrici strettamente correlate alle attività umane, aiuta a comprendere il ciclo completo di Salmonella spp. permettendo in tal modo di valutare meglio il ruolo che l'ambiente riveste nella trasmissione dei patogeni enterici all'uomo. Nella regione Molise le attività di sorveglianza relative al comparto ambientale hanno mostrato una sierotipìa di molto superiore a quella rinvenuta nel settore umano, in linea con i dati nazionali e in contrasto con quanto accade nelle matrici alimentari e nell'uomo, nell'ambito delle quali prevale un certa omogeneità sierologica, con la solita predominanza delle specie endemiche associate ad infezione nell'uomo e negli animali. Tale riscontro suggerisce l'ipotesi che l'ambiente acquatico rappresenti l'habitat ottimale per un pool di serovars molto diversificato, dovuto probabilmente all'esistenza di numerosi e differenti serbatoi naturali (dagli animali da allevamento alle specie selvatiche).

In Molise tale ambiente non ha giocato fino ad ora un ruolo rilevante nella trasmissione dell'infezione, in quanto non si sono verificati isolamenti da alimenti vegetali, dalle acque potabili, né da quelle di balneazione; tuttavia, le conoscenze raggiunte hanno consentito di rappresentare in modo più esaustivo la molteplicità dei sierotipi che hanno circolato nel territorio regionale, confermando nel contempo la presenza "endemica" di determinati serovars significativi per le infezioni umane. Il presente studio vuole, pertanto, costituire un segnale d'allerta per le autorità competenti, che devono continuare a mantenere una stretta sorveglianza sulle matrici ambientali, nella consapevolezza del ruolo che queste matrici potrebbero rivestire in termini di impatto potenziale sulla salute pubblica.

\section{Ringraziamenti}

Si ringraziano per la cortese collaborazione:

Dr.ssa E.Barone*, dr.ssa R. Martone**, dr. A. De

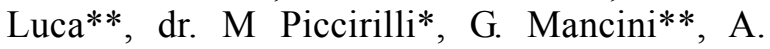
Mollichelli**, M.L. Vignone**.

*ARPA Molise, Dipartimento Provinciale di Isernia.

** ARPA Molise, Dipartimento Provinciale di Campobasso

Un ringraziamento particolare alle Dr.sse I. Luzzi ed E. Filetici

Dipartimento di Malattie Infettive, Parassitarie e Immunomediate

Reparto Malattie Batteriche, Gastroenteriche e Neurologiche

Istituto Superiore di Sanità (Roma) 


\section{BIBLIOGRAFIA}

1. APAT - IRSA. Metodi analitici per le acque. Vol. $3^{\circ}$ Edizione 29/2003.

2. Aulicino FA, Volterra L. Liquami di origine domestica: rischio sanitario e metodologie analitiche. Rapporto ISTISAN 2000; 00/17.

3. Cadrobbi P. La prevenzione e la terapia della salmonellosi umana. In atti del workshop su: Le salmonelle nell'ambiente e nell'uomo. Feltre (BL) settembre 2003; 28-32.

4. Caprioli A, Luzzi I, Lana S. Diagnostica ed epidemiologia delle zoonosi trasmesse da alimenti. IV Workshop Nazionale Enter-Net Italia - 04/ C4. novembre 2004 ; ISS Roma.

5. Caprioli A, Luzzi I, Lana S. Sorveglianza e prevenzione delle infezioni gastroenteriche. V Workshop Nazionale Enter-Net Italia - 05/C12, dicembre 2005; ISS Roma.

6. Cataldo C, Briancesco R, Bonadonna L. Acque di riuso: aspetti sanitari e tecnici correlati alla presenza di patogeni enterici. Rapporto Istisan 2001; 01/34.

7. D.Lvo $\mathrm{n}^{\circ} 99 / 92$ : Attuazione della direttiva 86/278/CEE concernente la protezione dell'ambiente, in particolare del suolo, nell'utilizzazione dei fanghi di depurazione in agricoltura. Supp. Ord. G.U. n`38, $15 / 02 / 1992$.

8. D.Lvo ${ }^{\circ} 152 / 99$ : Disposizioni sulla tutela delle acque dall'inquinamento e recepimento della direttiva 91/271/CEE concernente il trattamento delle acque reflue urbane e della direttiva 91/676/CEE relativa alla protezione delle acque dall'inquinamento provocato dai nitrati provenienti da fonti agricole, a seguito delle disposizioni correttive di cui al decreto legislativo 18 agosto $2000, n^{\circ} 258$. Supp. Ord. G.U. n 246 , 20/10/2000.

9. Galetta P, Luzzi I, Filetici E, Dionisi AM, Benedetti I, Arena S, et al. Enter-Net: sorveglianza delle infezioni da patogeni enterici. Isolamenti di Salm.spp. da infezioni umane in Italia nel 2003. In: ISTISAN- $\mathrm{n}^{\circ} 7-8$ 18:3-8. luglio/agosto 2005.

10. Graziani C, Galetta P, Busani L, et al. Infezioni da Salmonella: diagnostica, epidemiologia e sorveglianza.Rapporto ISTISAN 2005; 05/27.

11. Grazioli D. Le Salmonelle nelle acque ad uso umano del Feltrino. In atti del workshop su: Le salmonelle nell'ambiente e nell'uomo. Feltre (BL) settembre 2003; 21-3.

12. Luzzi I, Filetici E, Arena S, et al. Utilizzo della tipizzazione molecolare per l'individuazione della catena epidemiologica nello studio delle tossinfezioni alimentari da S.Typhimurium: descrizione di un episodio. In: Microbiologia Medica, 2001; 16:178.

13. Luzzi I. Le Salmonelle: tassonomia, ecologia e patogenicità. In atti del workshop su: Le salmonelle nell'ambiente e nell'uomo. Feltre (BL) settembre 2003; 5-8.

14. Manuppella A, Ricci N, Lucci A. Sorveglianza delle infezioni da Salmonella ed altri patogeni enterici nel Molise:1997/1998. In: Microbiologia Medica, 2000; 15: 337 - 42.

15. Manuppella A, Ricci N, Pede V. Il sistema di sorveglianza delle Salmonellosi nel Molise. In: Microbiologia Medica, 1994; 9: 32-3.

16. Popoff MY. Antigenic Formulas of the Salmonella Serovars. In: WHO Collaborating Centre for Reference and Research on Salmonella . Institut Pasteur, France $8^{\mathrm{a}}$ edition, 2001.

17. Rapporti annuali ENTER - NET 2003/2004; www.enternet@iss.it.

18. Ricci A, Mancini M, Cibin V, Busani L. In: Rapporto annuale Enter-Vet 2004. Istituto Zooprofilattico Sperimentale delle Venezie, ottobre 2005.

19. Ricci N, Manuppella A, Simeone R, et al. Su di un episodio di tossinfezione alimentare da Salmonella in una collettività scolare in provincia di Isernia. In: Giornale di malattie infettive e parassitarie,1982; 34: 1036-9.

20. Ricci N, Manuppella A, Laurelli T, De Vita G, Pede V, Meo R. Sierotipi di Salmonelle isolati dall'uomo, dagli alimenti e dall'ambiente in provincia di Isernia dal 1980 al 1989. In: Microbiologia Medica, 1991; 6: 147-51.

21. Ricci N, Manuppella A, Laurelli T, Durante M, Trillo P, Simeone R. Episodio di tossinfezione alimentare da gelato artigianale dovuto ad un sierotipo di Salmonella di raro riscontro. In: Igiene Moderna, 1990. 94: 240-44.

22. Ricci N, Manuppella A, Pede V, et al. Diffusione epidemica di S.Enteritidis nel Molise: 1989-1992. In: Microbiologia Medica, 1994; 9: 22-5.

23. Viel L, Ferro Milone NM, Bado L, Lazzarin I, Zannini G, Scalari F. Le Salmonelle negli animali della provincia di Belluno. In: Le Salmonelle nell'ambiente e nell'uomo. Feltre (BL); 2003; 13-18.

24. Zavanella M.Tipizzare le salmonelle. In: Fondazione Iniziative Zooprofilattiche e Zootecniche. Brescia, 2001.

\section{Annamaria Manuppella}

ARPA Molise,

Dipartimento Provinciale di Isernia

Via Giovanni Berta, Palazzo Provincia

86170 Isernia

Tel.: 086526994 - Fax: 0865414986

E-mail: isernia.dip@arpamolise.it 